\title{
Evaluation of the Quality of Life in Adult Cancer Survivors (QLACS) scale for early post-treatment breast cancer survivors
}

\author{
Stephanie J. Sohl, \\ Department of Medicine, Vanderbilt University School of Medicine, Sixth Floor, Suite 600, 2525 \\ West End Avenue, Nashville, TN 37203-1738, USA \\ Beverly Levine, and \\ Division of Public Health Sciences, Department of Social Sciences and Health Policy, Medical \\ Center Boulevard, Wake Forest School of Medicine, Winston-Salem, NC 27157-1063, USA \\ Nancy E. Avis \\ Division of Public Health Sciences, Department of Social Sciences and Health Policy, Medical \\ Center Boulevard, Wake Forest School of Medicine, Winston-Salem, NC 27157-1063, USA
}

Stephanie J. Sohl: Stephanie.Sohl@Vanderbilt.edu; Beverly Levine: blevine@wakehealth.edu; Nancy E. Avis: navis@wakehealth.edu

\section{Abstract}

Purpose-The end of primary treatment for cancer patients is increasingly recognized as an important time of adjustment that may impact quality of life (QoL). A psychometrically sound QoL instrument that assesses the mix of acute and longer-term concerns present during this unique time has not yet been identified. This article evaluates the Quality of Life in Adult Cancer Survivors (QLACS) scale, originally developed for long-term ( 25 years) cancer survivors, as an appropriate QoL measure for this transition period.

Methods-Psychometric properties of the QLACS were evaluated in a sample of post-treatment breast cancer survivors 18-24 months post-diagnosis. This observational study consisted of women $(n=552)$ aged 25 years and older (mean $=55.4$ years) who were diagnosed with stage $\mathrm{I}$, II, or III breast cancer. The 47 items of the QLACS comprise 12 domains: seven domains are generic, and five are cancer specific.

Results-The QLACS demonstrated adequate internal consistency (Cronbach's alpha for the 12 domains ranged from 0.79 to 0.91 ) and good convergent and divergent validity (assessed by comparison with the Functional Assessment of Cancer Therapy and other measures).

Conclusions-The QLACS appears to be consistent with other widely accepted measures in capturing QoL, while also allowing for more inclusive measurement of specific issues relevant to post-treatment cancer survivors. These data, in addition to previous data supporting use of the QLACS across different cancer sites, suggest that the QLACS is a promising comprehensive QoL measure appropriate for breast cancer survivors transitioning off active treatment.

(c) Springer International Publishing Switzerland 2014

Correspondence to: Nancy E. Avis, navis@wakehealth.edu. 


\section{Keywords}

Cancer; Oncology; Quality of life; Survivorship; Measurement

\section{Introduction}

Breast cancer mortality rates in the United States have significantly declined since 1975 [1] due in large part to improvements in therapy [2]. As a result of these declines, and in combination with an aging population, the number of breast cancer survivors has grown and is projected to continue increasing. There are approximately 2.9 million women living with breast cancer in the USA as of 2011, and $89.2 \%$ of the estimated 232,670 women diagnosed with breast cancer in 2014 are projected to be alive 5 years after diagnosis [3]. As defined by the National Cancer Institute, "a person is considered to be a survivor from the time of diagnosis until the end of life" (www.cancer.gov/dictionary). As breast cancer survivors live many years post-cancer diagnosis, quality-of-life $(\mathrm{QoL})$ issues related to continuing symptoms or problems among cancer survivors have become increasingly important [4].

To address an increased emphasis on QoL, a large number of cancer-specific QoL measures have been developed [5, 6]. Commonly used cancer-specific QoL measures, such as the Functional Assessment of Cancer Therapy-Breast (FACT-B; [7]) and the European Organization for Research and Treatment of Cancer (EORTC) Breast Cancer-Specific Quality of Life Questionnaire (QLQ-BR23; [8]), were designed primarily for cancer survivors in active treatment and have a predominance of items relevant to immediate effects of cancer diagnosis and treatment (e.g., nausea, shortness of breath). The impact of cancer on QoL during the acute diagnostic and treatment phases has been well studied [e.g., 9-11].

Quality of life among long-term cancer survivors (i.e., 25 years post-treatment) is also a growing area of study that aims to identify the long-term and late effects of cancer treatment. These studies typically compare cancer survivors to healthy controls using a generic QoL measure such as the SF-36 [4, 12, 13]. However, such generic measures do not characterize issues relevant to post-treatment cancer survivors such as cognitive problems, financial concerns, or fears of recurrence.

The Quality of Life in Adult Cancer Survivors (QLACS) scale was developed for long-term cancer survivors in response to limitations of QoL scales that focused mostly on acute diagnostic- and treatment-related effects and to limitations of generic measures to assess QoL in cancer survivors [14]. Scale development was based on research among survivors of cancers at various sites (breast, bladder, colorectal, gynecologic, head and neck, and prostate) who were at least 5 years post-diagnosis. The QLACS was designed to measure seven domains that capture quality-of-life components that reflect issues of importance to cancer survivors (e.g., cognitive problems, fatigue, sexual problems), but purposely do not mention cancer to allow comparison between survivors and the general population. The QLACS developers thus refer to these domains as generic. In addition, five of the QLACS domains are cancer specific and refer to cancer-related concerns. A review of QoL instruments for long-term breast cancer survivors noted that the QLACS had good 
psychometric properties such as high internal consistency, high validity, and good responsiveness compared to other QoL instruments used in this population [5]. The psychometric properties of the QLACS scale were further evaluated in long-term (8-year) breast cancer survivors [15] and demonstrated good test-retest reliability, high internal consistency, high responsiveness to life changes, and good validity.

To date, QoL research has focused primarily on cancer survivors during active treatment and long-term survivors. However, the time of transition immediately following completion of adjuvant treatment (i.e., chemotherapy, radiation) when patients lose ready access to health care and are reestablishing new normal life patterns has not been well studied. Researchers and clinicians are increasingly interested in this time period, which is considered to be particularly stressful due to new issues that may develop such as financial worries, concern about the family's future, fear of recurrence, lack of preparedness for lingering side effects of treatment (e.g., pain, fatigue, sexual problems, cognitive difficulties, psychological distress), and/or possible changes in social support [16-20]. How one handles the stress experienced during this time period is related to long-term adjustment [21-23]. Cancer survivors may also begin to report positive outcomes of their disease that result from the process of coping with this challenging transition. For example, women have reported better personal relationships, a change in priorities, and/or an increased appreciation for life [2426], which may be perceived as benefits resulting from being diagnosed with breast cancer.

A recent review of QoL scales for cancer survivors [5] identified a particular need for psychometrically sound QoL instruments appropriate for cancer survivors who are postprimary treatment and 1-5 years post-diagnosis, in order to capture the mix of symptoms that arise during treatment and new issues that develop during the transition off primary treatment. Although originally developed for long-term cancer survivors, the QLACS targets issues pertinent as well to those new to post-treatment and thus may also be appropriate for early-transition cancer survivors. However, the validity of the QLACS for this survivorship period has not been evaluated. The present study was designed to address the gap in measures suitable for early-transition survivors by evaluating some of the psychometric properties of the QLACS in a sample of breast cancer survivors who were 18-24 months post-diagnosis.

\section{Methods}

\section{Setting and population}

The data reported in these analyses were derived from an observational study conducted among women aged 25 years and older who were newly diagnosed with stage I, II, or III breast cancer. The study was developed to evaluate age differences in adjustment to breast cancer. Study design has been previously described [27-29]. Briefly, eligibility criteria included first time breast cancer diagnosis, cancer stage I-III, completion of baseline survey within 8 months of diagnosis, at least 18 years of age (although no one in the study was younger than age 25), and ability to read and understand English. Recruitment was conducted at Memorial Sloan Kettering Cancer Center and the University of TexasSouthwestern Center for Breast Care from 2002 to 2006. Women were recruited through 
hospital clinics and advertisements and initially screened by chart review or telephone for eligibility.

Eligible women were mailed a baseline questionnaire to complete and return to the Coordinating Center at Wake Forest University. Baseline questionnaires were completed within 8 months of diagnosis. Follow-up surveys were sent at 6,12, and 18 months following completion of the baseline survey. The analyses below focus on responses to the QLACS at the last follow-up survey when respondents were post-primary treatment and between 18 and 24 months post-diagnosis. To evaluate convergent and divergent validity of the QLACS, we selected additional measures to include in analyses because they assessed constructs similar to the various QLACS domains. These other measures were administered at all surveys. All sites obtained approval from their Institutional Review Boards.

\section{Measures}

Quality of Life in Adult Cancer Survivors (QLACS)—The QLACS contains 47 items scored on a 1 (never)-to-7 (always) scale [14]. The 47 items are grouped into 12 domains: seven are referred to as generic and five as cancer specific. Generic domains address issues relevant to cancer survivors, but do not ask specifically about cancer, and can be answered by general population samples: negative feelings, positive feelings, cognitive problems, sexual problems, physical pain, fatigue and social avoidance. A generic summary score (QLACS Generic Summary) is calculated by adding the seven constituent domain scores (reversing the score for positive feelings) so that a lower score (i.e., more frequent reports of "never") corresponds to higher QoL [14]. Cancer-specific domains relate specifically to having had cancer and include financial problems, family-related distress, distress about recurrence, appearance concerns, and benefits of having cancer. A cancer-specific summary score (QLACS Cancer-Specific Summary) is calculated by adding the constituent domain scores of all but the benefits domain. The benefits domain did not load with the other cancer-specific domains in scale development factor analyses and is reported separately from the Cancer-Specific Summary score [14].

Functional Assessment of Cancer Therapy Breast (FACT-B) was used as a comparison measure because it is one of the most commonly used measures of QoL for cancer survivors during active treatment. Also, it is often used for cancer survivors who have recently completed treatment since there is no measure specifically designed to assess QoL during the transition period of interest [7]. The FACT-B consists of the FACT General (FACT-G) and the Breast-Specific Concerns subscale (BSC). The FACT-G [30] is a cancer-specific QoL measure containing a total of 26 items consisting of four subscales: physical wellbeing, functional well-being, emotional well-being, and social/family wellbeing. A total FACT-G score is calculated by summing the subscales $(a=0.91)$. The BSC is a separate breast cancer-specific subscale that contains nine items focusing on breast cancer $(\alpha=0.69)$. All items are rated on a 5-point Likert scale and the respondent is asked to refer to the past seven days. Higher scores reflect better QoL.

Medical Outcomes Short-Form Health Survey ( $S F-36$ ) is a 36-item measure of health status for use in a general population [31]. The SF-36 consists of eight domains and two summary 
scores: the Physical Component Summary (PCS) and Mental Component Summary (MCS) score. Higher scores on the SF-36 indicate a better health status.

Global Quality of Life-A 100-mm visual analog scale was used to assess global QoL. The visual analog scale is a validated, single-item measure that asks respondents to rate their overall QoL in the past 2 weeks on a scale of 1-100 where 1 represents the lowest possible QoL and 100 represents the highest QoL. It has shown excellent validity and test-retest reliability [32].

Symptoms-Women completed a 39-symptomlist as used in the Women's Health Initiative [33] and based on the Breast Cancer Prevention Trial Symptom scale [34] consisting of a wide range of symptoms that can be a result of cancer treatment and aging, including symptoms that are physical (e.g., mouth ulcers, headaches, diarrhea, nausea, joint pains), emotional/psychological (e.g., mood changes, feeling depressed, forgetfulness), menopausal (e.g., hot flashes, vaginal dryness), and related to role difficulties (e.g., lowered work performance, avoidance of social affairs, decreased efficiency). All symptoms were rated on a four-point ordinal scale ranging from none to severe. Symptom scores were summed to provide an overall score with higher scores indicating higher levels of symptoms. In addition, scores for the fatigue and social avoidance items and a composite score of cognitive symptoms (mean of scores for forgetfulness and difficulty concentrating) were used in the analyses.

Self-assessed health—Respondents rated their health on the widely used one-item measure asking about their overall health today on a five-point scale (excellent, very good, good, fair, poor). Self-assessed health has been shown to be highly related to both morbidity and mortality [35].

Psychosocial variables-The Illness Intrusiveness Ratings Scale assessed the degree to which breast cancer diagnosis and treatment affected thirteen life areas: health, diet, paid work, active recreation, passive recreation, financial situation, relationship with spouse, sex life, family relations, other social relations, self-expression, religious expression, and community [36]. Total scores can range from 13 to 91 [37], with higher scores indicating more intrusiveness. The internal consistency for the scale in this sample was $a=0.90$. Perceived attractiveness was measured by three items from the Lasry body image scale that assess how a woman perceives her attractiveness [38]. Women were asked to rate their agreement with three statements: feeling attractive to others, attractiveness has changed due to surgery, and fear of being unattractive sexually. Scores can range from 3 to 15 , and higher scores indicate greater perceived attractiveness (coefficient alpha in our sample was 0.54).

Post-traumatic Growth, or positive outcomes from coping with a life challenge, was assessed with the Post-traumatic Growth Inventory developed by Tedeschi and Calhoun [39], with demonstrated validity [40] and high internal consistency in our sample $(\alpha=0.94)$. Higher scores indicate more growth.

Sociodemographic variables-We considered age, race, the ability to pay for basics, education, marital/partner status, and presence of children under age 18 in the home in our analyses. 
Cancer-related variables-The following variables were obtained from medical chart review performed at the end of primary treatment: cancer stage at diagnosis (I, II, or III), type of surgery (lumpectomy or mastectomy), radiation therapy (yes/no), chemotherapy (yes/no), hormonal therapy (yes/no), and time since diagnosis.

Analyses-Our analytic sample consisted of women who had scores on all of the QLACS domains at the 18-month follow-up $(n=552)$. Psychometric properties of the QLACS domains were examined in terms of floor and ceiling effects and internal consistency, as measured by Cronbach's alpha, for each domain and summary score. To assess convergent validity of the QLACS, we computed Pearson's correlation coefficients for the associations between QLACS domain and summary scores and other measures of related constructs that were administered at the 18-month follow-up.

We hypothesized that the QLACS Generic Summary score would be highly (i.e., $|r|>0.50$ ) negatively correlated with the other general QoL measures (i.e., FACT-G, SF-36, Global QoL), and the QLACS Cancer-Specific Summary would have a stronger correlation with the FACT-BSC than with general QoL measures, as found in previous analyses of long-term cancer survivors [14]. In addition, we expected that both QLACS summary scores (generic and cancer specific) would be positively related to total symptoms, self-assessed health, and illness intrusiveness scores. We also hypothesized that domain-specific QLACS scores would correlate highly with specific similar domains or items of measures of related constructs (e.g., QLACS physical pain domain with the SF-36 bodily pain; QLACS appearance concerns with perceived attractiveness; and QLACS benefits with post-traumatic growth)

To further assess convergent and divergent validity of the QLACS, we computed Pearson's correlation coefficients for the associations between the subscales of the QLACS and the subscales of the FACT-B. We expected that the QLACS subscales would be highly negatively related to corresponding scales on the FACT-B (i.e., QLACS negative feelings with FACT-B emotional; QLACS physical pain and fatigue with FACT-B physical; QLACS sexual problems and social avoidance with FACT-B social; QLACS positive feelings and cognitive problems with FACT-B functional; and QLACS familyrelated distress and appearance concerns with FACT-B BSC) and reflect divergent validity with lower correlations with constructs not assessed by the FACT-B (i.e., financial problems, distress about recurrence, and benefits of having cancer). All analyses were conducted using SAS 9.3 .

\section{Results}

\section{Sample characteristics}

A total of 740 surveys were mailed to women deemed eligible from chart reviews or telephone screening; 653 women completed baseline surveys for a response rate of $88 \%$. Of these 653 women, 565 remained in the study at the 18-month follow-up (86.5\%). Thirteen of these women did not complete the QLACS for an analytic sample of 552 women. 
Table 1 shows baseline characteristics of the sample. The mean agewas $55.4(\mathrm{SD}=12.4)$, and a majority were married or partnered (73.4\%), and white (91.3\%). Over half of the women $(53.1 \%)$ were diagnosed with Stage I disease. Most participants reported previously receiving chemotherapy $(67.2 \%)$ and hormonal therapy $(74.5 \%)$. The average time since diagnosis at baseline was 4.5 months ( $\mathrm{SD}=1.3$ months; range 3 days to 7.4 months). At the 18-month follow-up, the average length of time since diagnosis was 22.6 months ( $\mathrm{SD}=1.4$ months). All participants had completed primary treatment by the 18-month follow-up.

\section{Characteristics of the QLACS}

Table 2 shows the mean scores, minimum and maximum values, floor and ceiling effects and Cronbach's alpha for each domain, and summary score of the QLACS. None of the domains or summary scores showed ceiling effects (i.e., concentration of scores at the maximum). Three domains (physical pain, social avoidance, and financial problems) showed floor effects, with $33.9,35.9$, and $48.9 \%$ of the sample, respectively, reporting the minimum value. Internal consistency was good for the 12 domains (a ranged from 0.79 to 0.91 ).

\section{Convergent and divergent validity}

Table 3 shows results of the convergent validity analyses (all correlations were significant at $p<.001)$. As hypothesized, the QLACS Generic Summary score was highly correlated in a negative direction with assessments of general QoL such as the FACT-G $(r=-0.84)$, the SF-36 MCS $(r=-0.74)$, and global QoL $(r=-0.67)$. We also found a high correlation with the total symptom score $(r=0.76)$ and illness intrusiveness $(r=0.64)$. The QLACS Generic Summary score was somewhat less correlated with the SF-36 PCS $(r=-0.40)$ and selfassessed health $(r=0.49)$. Further, as hypothesized, the QLACS Cancer-Specific Summary score was more strongly correlated with the FACT-BSC $(r=-0.73)$ than with the general QoL measures (e.g., SF-36), and this correlation was stronger than the QLACS Generic Summary and the FACT-BSC.

All of the Generic QLACS domain scores were correlated highly with their similar measures. For instance, the QLACS fatigue score correlated with the SF-36 vitality score ( $r$ $=-0.82$ ), and both the QLACS positive feelings and negative feelings domains were correlated highly with the SF-36 mental health domain score $(r=0.77$ and -0.74 , respectively). The QLACS cancer-specific domains of appearance problems and benefits were also correlated with related measures of perceived attractiveness and post-traumatic growth ( -0.61 and 0.67 , respectively). Table 4 shows the correlations between QLACS domains and the subscales of the FACT-B. All correlations in this table, with three subscripted exceptions, were significant at $p<.001$.

A majority of the QLACS domains showed higher convergent validity with FACT subscales that assessed related constructs (e.g., QLACS negative feelings and FACT-B emotional well-being) and divergent validity with those FACT subscales that assessed unrelated constructs (e.g., QLACS pain and FACT-B social well-being). In addition, two QLACS domains that demonstrated somewhat divergent validity with all subscales of the FACT-B included financial problems (all $r$ 's $<0.31$ ) and benefits of having cancer (all $r$ 's $<0.22$ ). 


\section{Discussion}

The current study reports some psychometric properties of the QLACS for QoL assessment in breast cancer survivors who are 18-24 months post-diagnosis. Specifically, the QLACS demonstrated adequate internal consistency and good convergent and divergent validity in this sample. The QLACS appears to capture QoL as similarly as other widely accepted QoL measures, while also allowing for an expanded measurement of issues relevant to posttreatment cancer survivors, such as financial issues and concerns about recurrence. Thus, the QLACS is a promising answer to the call for QoL measures for cancer survivors transitioning off active treatment [5].

Results further supported the hypothesis that the QLACS Generic Summary score would exhibit stronger convergent validity with measures designed to assess general QoL and that the QLACS Cancer-Specific Summary would have a stronger correlation with the FACTBSC. The weakest correlation was between the SF-36 PCS and the QLACS Generic Summary score $(-0.40)$, which is not surprising because the SF-36 PCS emphasizes physical function, a construct that is not a part of the QLACS. Results also showed that the QLACS domain-specific scores exhibited convergent validity with other measures designed to assess similar constructs.

In addition, the QLACS primarily demonstrated convergent and divergent validity with the FACT-B as hypothesized, with two QLACS subscales illustrating that they assessed more divergent constructs (financial problems, benefits of having cancer). However, the QLACS subscale of distress about recurrence was moderately correlated with the FACT-B BSC, although this was not hypothesized. Thus, the QLACS assesses QoL similar to the FACT-B with the added benefit that the QLACS allows measurement of specific, concrete issues relevant to many cancer survivors not assessed by the FACT. For example, although many of the QLACS cancer-specific domains are correlated with the FACT-BSC, the FACT-BCS is a more global assessment. The QLACS allows one to specifically assess differences in adjustment to issues such as distress about recurrence or appearance concerns.

\section{Limitations and future directions}

One limitation of this study is the sociodemographic homogeneity of the sample. Further, because these analyses were conducted on study data not specifically designed to assess psychometric properties of the QLACS (hence, they are secondary analyses), the psychometric properties presented are not exhaustive and should continue to be evaluated. However, these limitations are accompanied by the strengths of the strong response and retention rates. Future research may further explore variability in how women adjust to breast cancer by QLACS domain. This research would lead to a more detailed understanding of quality of life and could inform targeting of interventions.

\section{Conclusion}

In summary, the QLACS is a reliable and valid assessment of general QoL and cancerspecific domains in early-transition breast cancer survivors. The QLACS appears to capture QoL similar to widely accepted QoL measures (e.g., FACT-B), while also allowing for a 
more specific measurement of issues relevant to post-treatment cancer survivors. Thus, these data in addition to previous data supporting use of the QLACS across different cancer sites [14] suggest that the QLACS is a promising comprehensive measure of QoL for early posttreatment breast cancer survivors. The QLACS could be particularly useful for longitudinal studies that begin assessment of cancer survivors during this transition period and continue through longer-term survivorship.

\section{Acknowledgments}

Research reported in this publication was supported by the National Cancer Institute Grant R25 CA122061, Building Interdisciplinary Research Careers in Women's Health (BIRCWH) Scholar Program (2K12HD043483-11), and Department of Defense DOD DAMD17-01-1-0447 (PI: Avis). The content is solely the responsibility of the authors and does not necessarily represent the official views of the National Institutes of Health or the Department of Defense.

\section{References}

1. Berry DA, Cronin KA, Plevritis SK, Fryback DG, Clarke L, Zelen M, et al. Effect of screening and adjuvant therapy on mortality from breast cancer. The New England Journal of Medicine. 2005; 353(17):1784-1792. [PubMed: 16251534]

2. Bleyer A, Welch HG. Effect of three decades of screening mammography on breast-cancer incidence. The New England Journal of Medicine. 2012; 367(21):1998-2005. [PubMed: 23171096]

3. Howlader, N.; Noone, AM.; Krapcho, M.; Garshell, J.; Miller, D.; Altekruse, SF., et al. SEER Cancer Statistics Review, 1975-2011. Bethesda, MD: National Cancer Institute; 2014. Retrieved April 21, 2014 from: http://seer.cancer.gov/csr/1975_2011/, based on November 2013 SEER data submission.

4. Ganz PA, Rowland JH, Desmond K, Meyerowitz BE, Wyatt GE. Life after breast cancer: Understanding women's health-related quality of life and sexual functioning. Journal of Clinical Oncology. 1998; 16(2):501-514. [PubMed: 9469334]

5. Pearce NJM, Sanson-Fisher R, Campbell HS. Measuring quality of life in cancer survivors: A methodological review of existing scales. Psycho-oncology. 2008; 17(7):629-640. [PubMed: 17973235]

6. Chopra I, Kamal KM. A systematic review of quality of life instruments in long-term breast cancer survivors. Health and Quality of Life Outcomes. 2012; 10:14. [PubMed: 22289425]

7. Brady MJ, Cella DF, Mo F, Bonomi AE, Tulsky DS, Lloyd SR, et al. Reliability and validity of the Functional Assessment of Cancer Therapy-Breast (FACT-B) quality of life instrument. Journal of Clinical Oncology. 1997; 15:974-986. [PubMed: 9060536]

8. Sprangers MA, Groenvold M, Arraras JI, Franklin J, te Velde A, Muller M, et al. The European Organization for Research and Treatment of Cancer breast cancer-specific quality-of-life questionnaire module: first results from a three-country field study. Journal of Clinical Oncology. 1996; 14(10):2756-2768. [PubMed: 8874337]

9. Kroenke CH, Kwan ML, Neugut AI, Ergas IJ, Wright JD, Caan BJ, et al. Social networks, social support mechanisms after breast cancer diagnosis. Breast Cancer Research and Treatment. 2013; 139:515-527. [PubMed: 23657404]

10. Kwan ML, Ergas IJ, Somkin CP, Quesenberry CP Jr, Neugut AI, Hershman D, et al. Quality of life among women recently diagnosed with invasive breast cancer: The pathways study. Breast Cancer Research and Treatment. 2010; 123(2):507-524. [PubMed: 20140494]

11. Arora NK, Gustafson DH, Hawkins RP, McTavish F, Cella DF, Pingree S, et al. Impact of surgery and chemotherapy on the quality of life of younger women with breast carcinoma. Cancer. 2001; 92(5):98-1288.

12. Baker F, Haffer SC, Denniston M. Health-related quality of life of cancer and noncancer patients in medicare managed care. Cancer. 2003; 97(3):674-681. [PubMed: 12548610] 
13. Ganz PA, Desmond KA, Leedham B, Rowland JH, Meyerowitz BE, Belin TR. Quality of life in long-term, disease-free survivors of breast cancer: A follow-up study. Journal of the National Cancer Institute. 2002; 94(1):39-49. [PubMed: 11773281]

14. Avis NE, Smith KW, McGraw S, Smith RG, Petronis VM, Carver CS. Assessing quality of life in adult cancer survivors (QLACS). Quality of Life Research. 2005; 14(4):1007-1023. [PubMed: 16041897]

15. Avis NE, Ip E, Foley KL. Evaluation of the Quality of Life in Adult Cancer Survivors (QLACS) scale for long-term cancer survivors in a sample of breast cancer survivors. Health and Quality of Life Outcomes. 2006; 4:92. [PubMed: 17140438]

16. Ganz PA, Guadagnoli E, Landrum MB, Lash TL, Rakowski W, Silliman RA. Breast cancer in olderwomen:Quality of life and psychosocial adjustment in the 15 months after diagnosis. Journal of Clinical Oncology. 2003; 21(21):4027-4033. [PubMed: 14581426]

17. Klein D, Mercier M, Abeilard E, Puyraveau M, Danzon A, Dalstein V, et al. Long-term quality of life after breast cancer: A French registry-based controlled study. Breast Cancer Research and Treatment. 2011; 129(1):125-134. [PubMed: 21340477]

18. Lee ES, Lee MK, Kim SH, Ro JS, Kang HS, Kim SW, et al. Health-related quality of life in survivors with breast cancer 1 year after diagnosis compared with the general population: A prospective cohort study. Annals of Surgery. 2011; 253(1):101-108. [PubMed: 21294288]

19. Taira N, Shimozuma K, Shiroiwa T, Ohsumi S, Kuroi K, Saji S, et al. Associations among baseline variables, treatment-related factors and health-related quality of life 2 years after breast cancer surgery. Breast Cancer Research and Treatment. 2011; 128(3):735-747. [PubMed: 21681445]

20. National Research Council. From cancer patient to cancer survivor: Lost in transition. Washington, D.C.: The National Academies Press; 2005.

21. Garofalo JP, Choppala S, Hamann HA, Gjerde J. Uncertainty during the transition from cancer patient to survivor. Cancer Nursing. 2009; 32(4):E8-E14. [PubMed: 19444082]

22. Hulbert-Williams N, Neal R, Morrison V, Hood K, Wilkinson C. Anxiety, depression and quality of life after cancer diagnosis: What psychosocial variables best predict how patients adjust? Psycho-oncology. 2011; 21:857-867. [PubMed: 21695744]

23. Carver CS, Antoni MH. Finding benefit in breast cancer during the year after diagnosis predicts better adjustment 5 to 8 years after diagnosis. Health Psychology. 2004; 23(6):595-598. [PubMed: 15546227]

24. Cordova MJ, Cunningham LL, Carlson CR, Andrykowski MA. Posttraumatic growth following breast cancer: A controlled comparison study. Health Psychology. 2001; 20(3):176-185. [PubMed: 11403215]

25. Sears SR, Stanton AL, Danoff-Burg S. The yellow brick road and the emerald city: Benefit finding, positive reappraisal coping and posttraumatic growth in women with early-stage breast cancer. Health Psychology. 2003; 22(5):487-497. [PubMed: 14570532]

26. Danhauer SC, Case LD, Tedeschi R, Russell G, Vishnevsky T, Triplett K, et al. Predictors of posttraumatic growth in women with breast cancer. Psycho-Oncology. 2013; 22(12):2676-83. [PubMed: 24136875]

27. Sohl SJ, Levine B, Case LD, Danhauer SC, Avis NE. Trajectories of illness intrusiveness domains following a diagnosis of breast cancer. Health Psychology. 2014; 33(3):232-41. [PubMed: 23668843]

28. Avis NE, Levine B, Naughton MJ, Case DL, Naftalis E, Van Zee KJ. Explaining age-related differences in depression following breast cancer diagnosis and treatment. Breast Cancer Research and Treatment. 2012; 136(2):581-591. [PubMed: 23053661]

29. Avis NE, Levine B, Naughton MJ, Case L, Douglas, Naftalis E, et al. Age-related longitudinal changes in depressive symptoms following breast cancer diagnosis and treatment. Breast Cancer Research and Treatment. 2013; 139:199-206. [PubMed: 23588951]

30. Cella DF, Tulsky DS, Gray G, Sarafian B, Lloyd S, Linn E, et al. The Functional Assessment of Cancer Therapy (FACT) scale: Development and validation of the general measure. Journal of Clinical Oncology. 1993; 11:570-579. [PubMed: 8445433]

31. Ware JE, Sherbourne CD. The MOS 36-item short-form health survey (SF-36). I. Conceptual framework and item selection. Medical Care. 1992; 30(6):473-483. [PubMed: 1593914] 
32. De Boer AGEM, van Lanschot JJB, Stalmeier PFM, van Sandick JW, Hulscher JBF, de Haes JCJM, et al. Is a single-item visual analogue scale as valid, reliable and responsive as multi-item scales in measuring quality of life? Quality of Life Research. 2004; 13(2):311-320. [PubMed: 15085903]

33. Barnabei VM, Cochrane BB, Aragaki AK, Nygaard I, Williams RS, McGovern PG, et al. Menopausal symptoms and treatment-related effects of estrogen and progestin in the Women's Health Initiative. Obstetrics and Gynecology. 2005; 105(5 Pt 1):1063-1073. [PubMed: 15863546]

34. Ganz PA, Day R, Ware JE Jr, Redmond C, Fisher B. Base-line quality-of-life assessment in the national surgical adjuvant breast and bowel project breast cancer prevention trial. Journal of the National Cancer Institute. 1995; 87(18):1372-1382. [PubMed: 7658498]

35. McDowell, I. Measuring health: A guide to rating scales. 3rd ed.. New York: Oxford University Press; 2006.

36. Devins GM, Binik YM, Hutchinson TA, Hollomby DJ, Barreé PE, Guttmann RD. The emotional impact of end-stage renal disease: Importance of patients' perception of intrusiveness and control. International Journal of Psychiatry in Medicine. 1983; 13(4):327-343. [PubMed: 6671863]

37. Devins GM. Using the illness intrusiveness ratings scale to understand health-related quality of life in chronic disease. Journal of Psychosomatic Research. 2010; 68(6):591-602. [PubMed: 20488277]

38. Lasry JC, Margolese RG, Poisson R, Shibata H, Fleischer D, Lafleur D, et al. Depression and body image following mastectomy and lumpectomy. Journal of Chronic Diseases. 1987; 40(6):529-534. [PubMed: 3597656]

39. Tedeschi RG, Calhoun LG. The posttraumatic growth inventory: Measuring the positive legacy of trauma. Journal of Traumatic Stress. 1996; 9(3):455-471. [PubMed: 8827649]

40. Brunet J, McDonough MH, Hadd V, Crocker PRE, Sabiston CM. The posttraumatic growth inventory: An examination of the factor structure and invariance among breast cancer survivors. Psycho-oncology. 2010; 19(8):830-838. [PubMed: 19862767] 
Table 1

Baseline characteristics of the total sample $(n=552)$

\begin{tabular}{|c|c|c|}
\hline \multirow[t]{2}{*}{ Characteristic } & \multicolumn{2}{|c|}{ Total sample } \\
\hline & $N$ & $(\%)$ \\
\hline Age in years $(\mathrm{M}[\mathrm{SD}])$ & 552 & $55.4(12.4)$ \\
\hline Race: white & 504 & $(91.3)$ \\
\hline \multicolumn{3}{|l|}{ Ability to pay for basics } \\
\hline Very hard & 15 & $(2.7)$ \\
\hline Somewhat hard & 77 & $(14.0)$ \\
\hline Not hard & 460 & $(83.3)$ \\
\hline Graduated college & 351 & $(63.6)$ \\
\hline Married or partnered & 405 & (73.4) \\
\hline Have children under 18 years living at home & 134 & $(24.3)$ \\
\hline \multicolumn{3}{|l|}{ Stage } \\
\hline I & 293 & $(53.1)$ \\
\hline II & 213 & $(38.6)$ \\
\hline III & 46 & $(8.3)$ \\
\hline \multicolumn{3}{|l|}{ Surgery } \\
\hline Lumpectomy only & 349 & $(63.2)$ \\
\hline Mastectomy & 203 & $(36.8)$ \\
\hline Chemotherapy-yes & 371 & $(67.2)$ \\
\hline Radiation-yes & 401 & (72.6) \\
\hline Hormonal therapy—yes & 411 & $(74.5)$ \\
\hline Months since diagnosis (M [SD]) & 552 & $4.5(1.3)$ \\
\hline
\end{tabular}


Table 3

Correlations of the Quality of Life in Adult Cancer Survivors (QLACS) domains with concurrent validity measures at 18-month follow-up

\begin{tabular}{|c|c|c|}
\hline Subscale & Related measure & $r$ \\
\hline \multirow[t]{8}{*}{ Generic summary } & FACT-G & -0.84 \\
\hline & FACT-BSC & -0.61 \\
\hline & SF-36 MCS & -0.74 \\
\hline & SF-36 PCS & -0.40 \\
\hline & Total symptom score & 0.76 \\
\hline & Global quality of life & -0.67 \\
\hline & Illness Intrusiveness & 0.64 \\
\hline & Self-assessed health & 0.49 \\
\hline \multirow[t]{8}{*}{ Cancer-specific summary } & FACT-BSC & -0.73 \\
\hline & FACT-G & -0.47 \\
\hline & SF-36 MCS & -0.35 \\
\hline & SF-36 PCS & -0.29 \\
\hline & Total symptom score & 0.51 \\
\hline & Global quality of life & -0.36 \\
\hline & Illness intrusiveness & 0.55 \\
\hline & Self-assessed health & 0.47 \\
\hline \multicolumn{3}{|l|}{ Generic domains } \\
\hline Fatigue & SF-36 vitality & -0.82 \\
\hline Fatigue & Fatigue symptom & 0.71 \\
\hline Positive feelings & SF-36 mental health & 0.77 \\
\hline Negative feelings & SF-36 mental health & -0.74 \\
\hline Physical pain & SF-36 bodily pain & -0.71 \\
\hline Cognitive problems & Cognitive symptoms & 0.81 \\
\hline Sexual problems & Sex life item from the IIRS & 0.67 \\
\hline Social avoidance & SF-36 social role functioning & -0.60 \\
\hline Social avoidance & Social avoidance symptom & 0.69 \\
\hline \multicolumn{3}{|l|}{ Cancer domain } \\
\hline Appearance problems & Perceived attractiveness & -0.61 \\
\hline Benefits & Post-traumatic growth & 0.67 \\
\hline
\end{tabular}

Functional Assessment of Cancer Therapy general and breast cancer-specific concerns subscale (FACT-G, FACT-BSC) Medical outcomes shortform Health Survey (SF-36): Mental Component Summary Score (MCS), Physical Component Summary Score (PCS); Illness Intrusiveness Ratings Scale (IIRS)

All correlations were significant at $p<0.001$. 


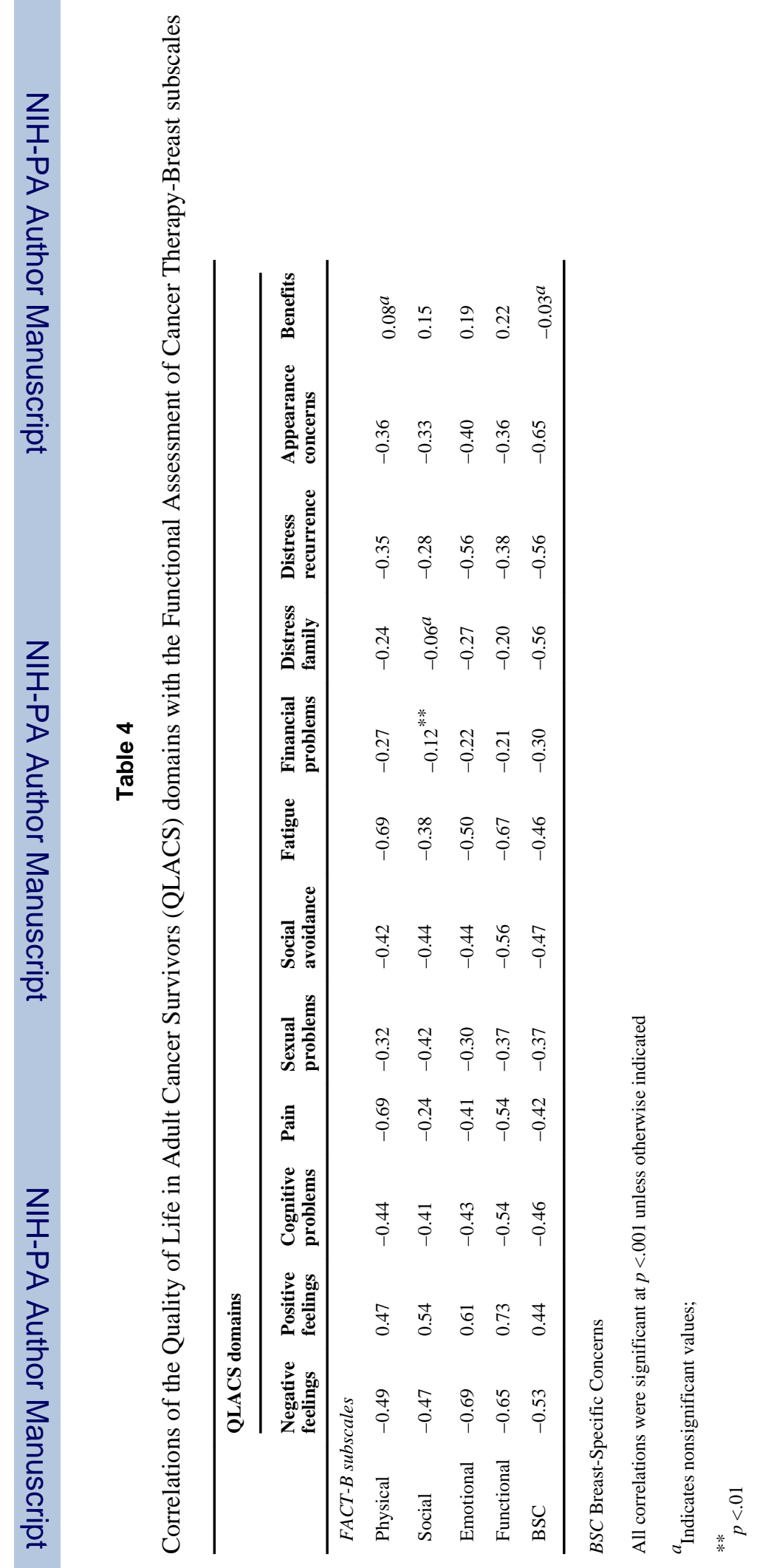

